\title{
Visual perception of collinearity
}

\author{
R. H. CUIJPERS, A. M. L. KAPPERS, and J. J. KOENDERINK \\ Helmholtz Instituut, Utrecht, The Netherlands
}

\begin{abstract}
In a metrical space, there exists an intimate relation between collinearity and parallelity. In particular, in a Riemannian space collinearity is just a special case of parallelity. Is this true for visual space as well? We investigated the visual perception of collinearity by having subjects align two bars in the horizontal plane at eye height. The distances of the bars from the subject and the angles at which they were placed were varied. We found deviations of up to $22^{\circ}$. The deviations of the left and right bars could be split into two independent components: namely, the sum and the difference of the deviations of the left and right bars. We found that the former depended only on the ratio between the distances of each bar from the subject, whereas the latter was largely independent of the positions of the bars. The difference in deviations corresponded to the deviation from parallelity. Compared with the results in the parallelity task (Cuijpers, Kappers, \& Koenderink, 2000b), the deviations from parallel were much smaller. As a consequence, the results of the two experiments cannot be described by the same Riemannian geometry. This indicates that the intrinsic geometry of visual space differs across tasks. This is conceivable if the intrinsic geometry of visual space is operationally defined.
\end{abstract}

Our visual perception of the environment provides sufficient information about the geometry of the environment for us to perform daily tasks such as navigation and grasping. However, the visual perception of the size of objects and the distance of these objects from the observer deviate considerably from veridical. Even so, the visual system must somehow ex tract the information necessary to perform the different tasks. The question is, which geometrical aspects are extracted and to what extent are they similar to the physical situation?

One way of looking at the problem is to assume that there exists a cognitive map of locations in the environment which is warped with respect to the physical layout. From this map all geometrical properties can be obtained subsequently. This map is usually referred to as visual space. This view involves two types of distortions: First, there is a mapping from physical to visual space that may be distorted, and second, the intrinsic structure - that is, the relations between points of visual space-may be distorted. Another way of looking at the problem is to assume that the geometrical aspects are obtained only from the visual input at the moment they are required. Mathematically, these two views need not be different because, in the latter case, visual space can be defined operationally. Physiologically, however, the situation is very different because, in the latter view, the existence of a cognitive map is unnecessary and the only requirement is to have methods for obtaining geometrical knowledge (similar to the perceptual systems of Gibson, 1966). A direct consequence is that different geometrical aspects (such as distance and

Correspondence should be addressed to A. M. L. Kappers, Helmholtz Instituut, Princetonplein 5, 3584 CC Utrecht, The Netherlands (email: a.m.1.kappers@phys.uu.nl). parallelity) need not be consistent with each other and that if visual space is defined operationally, the intrinsic structure will depend on the geometrical aspects in question. The bottom line is that the correct view is unknown, but both possibilities should be reckoned with.

Luneburg (1947) proposed a model, based on experiments of Hillebrand (1902) and Blumenfeld (1913), in which visual space is a hyperbolic space of constant negative curvature. In this model, the mapping from physical to visual space preserves egocentric direction and involves a distance function that expands the distance from the observer in the near field and contracts distance in the far field. Many researchers used the Luneburg model to determine the curvature of visual space (e.g., Battro, Pierro Netto, \& Rozestraten, 1976; Blank, 1961; Foley, 1972; Hardy, Rand, \& Rittler, 1951; Indow, 1991; Indow, Inoue, \& Matsushima, 1962a, 1962b; Indow \& Watanabe, 1984a, 1984b; Zajaczkowska, 1956a, 1956b), but the quantitative results were unsatisfactory because different methods produced different results. Furthermore, when experiments were conducted outside in broad daylight, the results could be described better by a simple distance relation (Battro, Reggini, \& Karts, 1978; Wagner, 1985). Direct tests of the assumption that visual space is a constantly curved space have revealed that the assumption is, at least partly, incorrect (e.g., Foley, 1963, 1972; Higashiyama, 1984; Koenderink, Van Doorn, \& Lappin, 2000).

In the literature many different distance relations have been proposed relating physical and visual space (e.g., Battro et al., 1978; Foley, 1980; Gilinsky, 1951; Wagner, 1985). Although these distance relations are adequate for the experiments from which they were derived, they are not consistent with each other. It appears that the form depends on the experimental method and the visual environment. The large influence of the visual context has been shown 
explicitly by Schoumans, Koenderink, and Kappers (2000) and Cuijpers, Kappers, and Koenderink (2001).

The most far-reaching assumption in the Luneburg model is that visual space is a metrical space of constant (negative) curvature. Blank (1958) showed that, theoretically, a sense of length ordering and a sense of alignment (or collinearity) are sufficient for the existence of some metric. Luneburg's assumption that visual space is isomorphic to a Riemannian metric, however, is much more restrictive because even when the constant curvature condition is relaxed, it still implies that concepts such as parallelity, collinearity, and length ordering are related to each other in a unique way. This can be tested experimentally because if visual space is Riemannian, the intrinsic structure of visual space should be the same when based on a parallelity or collinearity task.

The concept of parallelism in Riemannian spaces is somewhat different from that in Euclidean geometry. For example, on the surface of a sphere (elliptic geometry), great circles are equivalent to straight lines, but these lines will always intersect. On the other hand, for a given line in hyperbolic geometry, infinitely many lines passing through a distinct point will not intersect it. Thus, global parallelism does not exist in general in Riemannian spaces. However, it is still possible to define parallelism locally. For example, on the surface of a sphere all meridians intersect the equator at right angles. The tangent vectors of the meridians at the equator are considered parallel in the sense of Levi-Civita (Stoker, 1969) with respect to the equator. For any Riemannian space, parallelism is defined by means of parallel transport: displacing a vector along some curve without changing its length and orientation (independent of the choice of coordinates). A special case arises when a curve parallel transports its own tangent vector. This is a nice way to define straight lines in curved spaces, the socalled geodesics. The tangent vectors of a geodesic are not only parallel but also collinear. In terms of visual space, this corresponds to two (or more) line elements that lie on a visually straight line.

In a previous study (Cuijpers et al., 2000b), subjects had to set a test bar parallel to a reference bar, both placed horizontally at eye height and rotating about the vertical axes through their centers. We found large deviations, up to $40^{\circ}$, that increased linearly with the separation angle, but varying the distance of the bars from the subject had no effect. It was argued that subjects could have ignored the distance at which the bars were placed and based their responses on monocular information only. In other words, the task might be inadequate for sampling the depth structure of visual space. When subjects are asked to align two bars, they have to attend to the distance of the bars from themselves even though the orientation of the bars themselves can be judged monocularly. If judgments of collinearity do depend on distance and judgments of parallelity do not, this implies that collinear bars do not need to appear parallel. Such a finding would rule out a Riemannian metric as a complete description of visual space because in a Riemannian metric collinear vectors are always parallel.
In this study, we investigated the concept of collinearity. The subjects' task was to align two bars in the horizontal plane at eye height so that both bars appeared to be tangent to an imaginary straight line connecting the centers of the bars. The distance of the bars from the subject and the angle at which they were placed were varied. The results can be compared directly to the parallelity task because the same experimental setup was used. When both bars are placed at an equal distance from the subject in the frontoparallel plane, the results can be compared directly with the frontoparallel horopters in the literature (e.g., Battro et al., 1976; Indow \& Watanabe, 1984a, 1988; Ogle, 1964; Shipley, 1959; Zajaczkowska, 1956a) by interpreting the bars as tangent vectors of a physically curved arc. By placing the bars at unequal distances from the subject and at different angles, we also obtained slanted horopters in front of the subject and to the left and right of the subject. Direct measurements of horopters outside the frontoparallel plane have not been reported in the literature.

\section{METHOD}

\section{Subjects}

Four subjects with an average age of 20 years participated in the experiment. The subjects were unfamiliar with the aims of the experiment and received no feedback about their performance. All subjects had normal vision or wore contact lenses. Visual acuity was tested with a Landoldt $\mathrm{C}$ chart and was found to be better than visus 1 (acuity is better than 5 arcmin). Stereo acuity was tested with a standard TNO-test (Walraven, 1975) and found to be better than 60 arcsec. All visual judgments were made binocularly.

\section{Experimental Setup}

The experimental room was a $6 \mathrm{~m} \times 6 \mathrm{~m}$ room, the walls of which were covered by black plastic sheeting in a random fashion: The plastic was wrinkled, creating cavities and protrusions of the order of $10 \mathrm{~cm}$ (Figure 1a). The subject was seated on a chair; the position of the head was fixed by means of a head and chin rest. The subject could only see the experimental setup through a "visor" with an aperture that limited the visual field to a visual angle of about $10^{\circ}$ vertically and $140^{\circ}$ horizontally (Figure $1 \mathrm{~b}$ ). As a result, the floor and ceiling of the experimental room were invisible. The aperture was formed by bending a piece of black paper with a rectangular opening $(1.5 \mathrm{~cm}$ high and $40 \mathrm{~cm}$ wide) into a half-circle of $15 \mathrm{~cm}$ radius centered on the midpoint between the eyes. The black sheeting on the facing wall was shaped approximately as a ViethMüller circle of 5-m radius. The purpose of the plastic sheeting and the horizontal aperture was to hide external references as much as possible.

\section{Stimuli}

Two identical sets of four differently sized bars were used as stimuli. Each bar consisted of a white metal rod and a yellow disk: The rod protruded from both sides of the center of the disk at a right angle (Figure 1c). The purpose of the disk was to provide additional information about the orientation of the bar. The rods had pointed tips with a top angle of $60^{\circ}$. The bars were mounted horizontally at eye height on thin, vertical black rods. The thin vertical rods could be attached to a motor that was operated by remote control, so that the bars could rotate about the vertical axes through their centers. Their orientations could be read off from a scale. Only the bars and the upper part of the thin vertical rods were visible to the subject. The bars of each set were scaled in size so that the visual angle was kept constant when the bars were placed at the appropriate distance 
a)

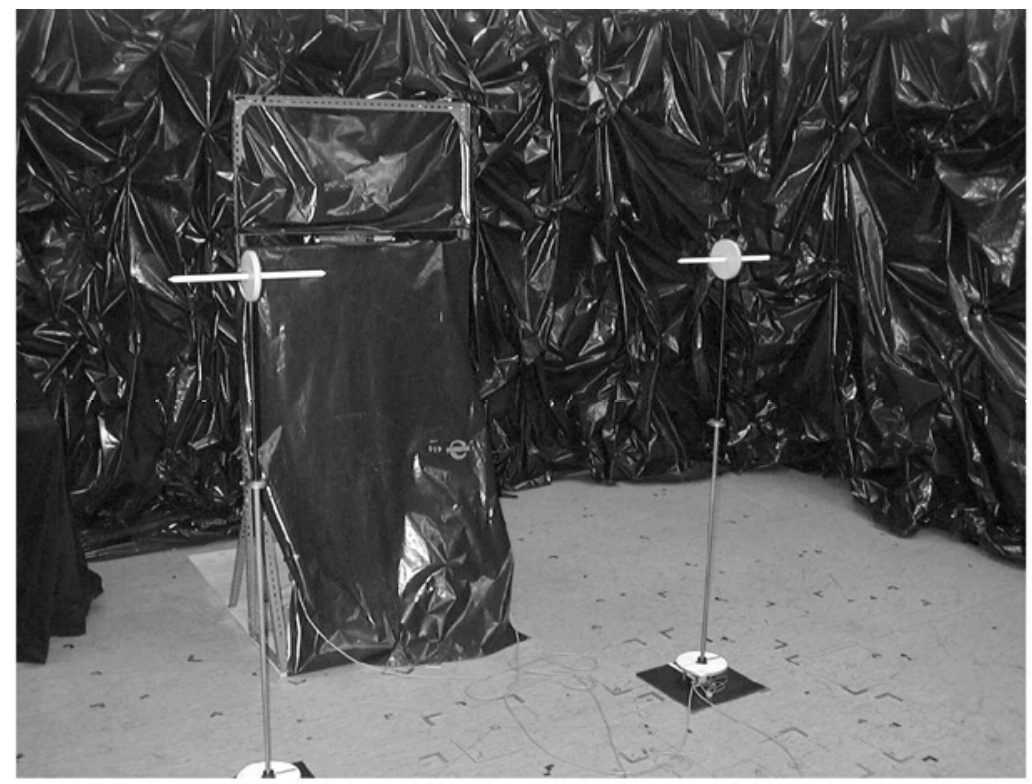

b)

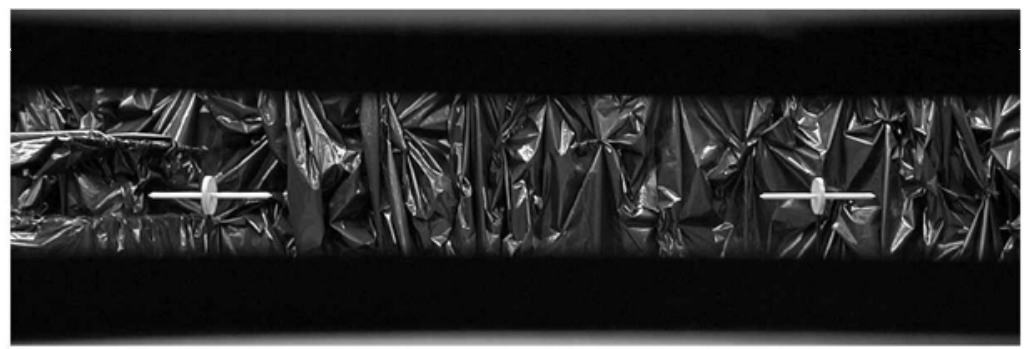

c)

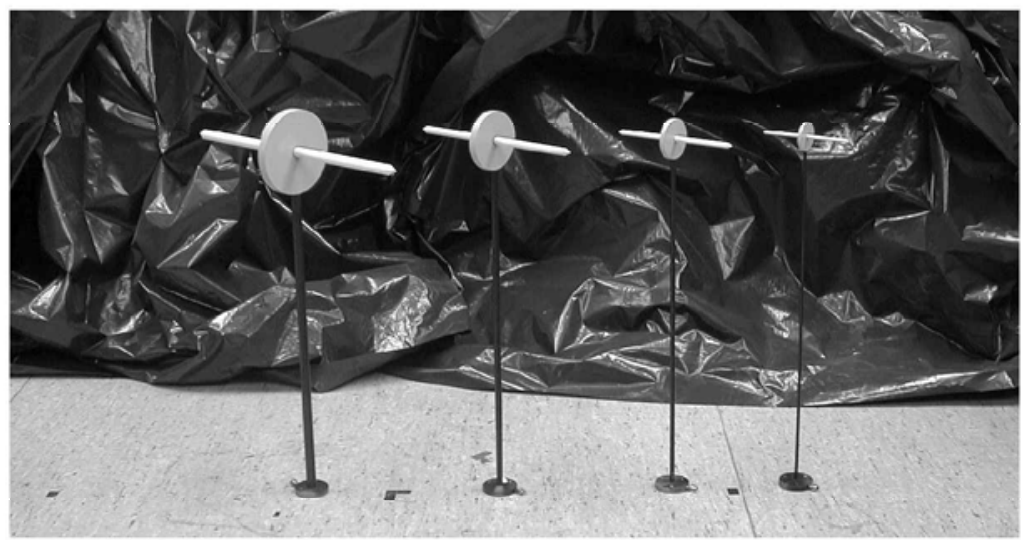

Figure 1. Pictures of the experimental setup: (a) The subject was seated behind a black plastic screen while viewing the two bars through a horizontal aperture. The walls were covered by wrinkled black plastic sheeting. (b) From the vantage point of the subject the field of view was limited to $10^{\circ}$ vertically and $140^{\circ}$ horizontally. (c) One of the two sets of bars. The bars of each set were scaled in size so that the subtended visual angle was constant when they were placed at the appropriate distance.

from the subject (Table 1). The width of the disks and the diameter of the vertical rods were equal to the diameter of the rods of the bar.

\section{Procedure}

In each trial a pair of bars was presented to the subject at varying distances (from the subject) and at different angles. The subjects' task was to set the two bars so that they looked collinear. In other words, subjects had to adjust the orientation of the two bars so that they appeared to be tangent to an imaginary straight line connecting the centers of the bars. The subjects were instructed by means of a drawing on a piece of paper.

The locations of the bars were selected from the intersections of lines of constant radius $(1.47,2.10,3.00$, and $4.31 \mathrm{~m})$ centered on the subject and the lines emanating from the subject at the polar an- 
Table 1

Dimensions of the Bars for Each Distance From the Subject

\begin{tabular}{lrrrr}
\hline & \multicolumn{4}{c}{ Distance $(\mathrm{m})$} \\
\cline { 2 - 5 } \multicolumn{1}{c}{ Dimension } & 1.47 & 2.10 & 3.00 & 4.31 \\
\hline Rod length $(\mathrm{mm})$ & 122 & 175 & 250 & 359 \\
Rod diameter (mm) & 5 & 7 & 10 & 14 \\
Disk diameter (mm) & 40 & 57 & 82 & 118 \\
\hline
\end{tabular}

gles $\psi$ of $\pm 30^{\circ}, \pm 15^{\circ}$, and $0^{\circ}$ (Figure 2 ). We did not use every possible combination, but rather, only the combinations with a separation angle $\zeta$ of $30^{\circ}$ or $60^{\circ}$. The separation angle is defined as the difference in polar angle between the bar to the left and to the right of the subject: $\zeta=\psi_{\text {left }}-\psi_{\text {right }}$. As a result, the following 4 combinations of the polar angles $\left(\psi_{\text {left }}, \psi_{\text {right }}\right)$ were used: $\left(30^{\circ},-30^{\circ}\right)$, $\left(30^{\circ}, 0^{\circ}\right),\left(15^{\circ},-15^{\circ}\right)$, and $\left(0^{\circ},-30^{\circ}\right)$. For each of these angle combinations, each bar was placed at all the possible distances, yielding 16 combinations of distances for the two bars.

The trials were presented in random order with the restrictions that only one bar at a time had to be moved between trials and that the right and left bars were moved alternately. These restrictions reduced the measuring time and made the experimenter's task considerably easier. When the subjects were satisfied with their settings, they signaled this to the experimenter and closed their eyes. After the orientations of the bars had been noted and the next trial had been set up, they were asked to rotate the bars an arbitrary amount with their eyes still closed. Once the experimenter was out of sight, the subjects received a signal and could open their eyes and start adjusting the bars. This was repeated until all 64 trials had been completed, albeit in two sessions. The whole procedure was measured three times for each subject and each time in a different random order. The measurements were conducted in sessions of about an hour. For 4 subjects the total measuring time amounted to $27 \mathrm{~h}$

The orientations of the bars are expressed as the angles $\phi_{\text {left }}$ and $\phi_{\text {right }}$ for the left and right bars, respectively (from the viewpoint of the subject). The counterclockwise direction is taken as positive and the $0^{\circ}$ orientation is parallel to the median line of the subject. Due to the symmetry of the bars, the angles $\phi$ have a periodicity of $180^{\circ}$. The deviations from veridical are defined as $\Delta \phi_{\text {left }}=\phi_{\text {left }}-\phi_{\text {veridical }}$ and $\Delta \phi_{\text {right }}=\phi_{\text {right }}-\phi_{\text {veridical }}$. Note that the veridical orientations are identical for two collinear bars. In addition, we were interested in the sum of the deviations of the left and right bars, denoted by $\sigma=$ $\Delta \phi_{\text {left }}+\Delta \phi_{\text {right }}$, and the difference in deviations, denoted by $\delta=$ $\Delta \phi_{\text {left }}-\Delta \phi_{\text {right }}$. As can be seen in Figure 3, the deviation difference $\delta$ is zero when the bars are physically parallel and the deviation sum $\sigma$ is zero when the orientations of the bars are cocircular- that is, mirrorsymmetric with respect to the normal of the connecting line. In the latter case the bars are tangent to a circular arc. In general, the orientations of the bars were neither parallel nor mirror-symmetric. In that case the deviation sum $\sigma$ corresponds to the symmetric part of the deviations, which is the deviation from collinearity, and the deviation difference $\delta$ corresponds to the mirror-symmetric part, which is the deviation from physically parallel.

\section{RESULTS}

Instead of showing all the data for all subjects and all combinations of polar angles at which the bars were placed, we have shown the data of 2 subjects for two combinations of angles. These results are representative in the sense that all systematic effects found for the other subjects and other angle combinations are also present in these data. The systematic trends for the other subjects and other combinations of polar angles will be shown in another form later on.

\section{Primary Analysis}

In Figure 4 the results are shown for Subjects T.D. and R.F. under the condition where the left bar was placed at $\psi_{\text {left }}=30^{\circ}$ and the right bar at $\psi_{\text {right }}=-30^{\circ}$. In each graph the coordinates are shown in units of meters. The location and the mean orientation of the bars (averaged over three measurements) are indicated by the solid lines. The centers of the bars are connected by a dashed line for each combination of the bar positions. The setting of the subject is veridical when the orientations of the bars are aligned with the dashed line. The deviation from veridical is shown numerically in degrees below each bar. The midpoint between the eyes of the subject is located in the origin. In each graph the combinations of the two bar locations are shown with a fixed ratio between the radial distances of the bars. For each subject, the ratios between the distance of the left and right bars are, from top to bottom, approximately $1, \sqrt{2}, 2$, and $2 \sqrt{2}$ in the left column and $1 / \sqrt{2}, 1 / 2$, and $1 / 2 \sqrt{2}$ in the right column.

Clearly, considerable deviations, of up to $22^{\circ}$, occur. Considering the fact that the standard deviation is about $2^{\circ}$ to $3^{\circ}$, it can be seen that within each graph (in other words, for a fixed distance ratio), the orientations of the left bar for each (absolute) distance are similar. The same is true for the right bar, although its orientation is in general different from that of the left bar. Hence, the difference in the orientation of the left and right bars (which equals the difference between the deviations $\delta$ ) is approximately constant for a fixed distance ratio. Moreover, a comparison between the graphs reveals that this difference is approximately independent of the distance ratio as well. For

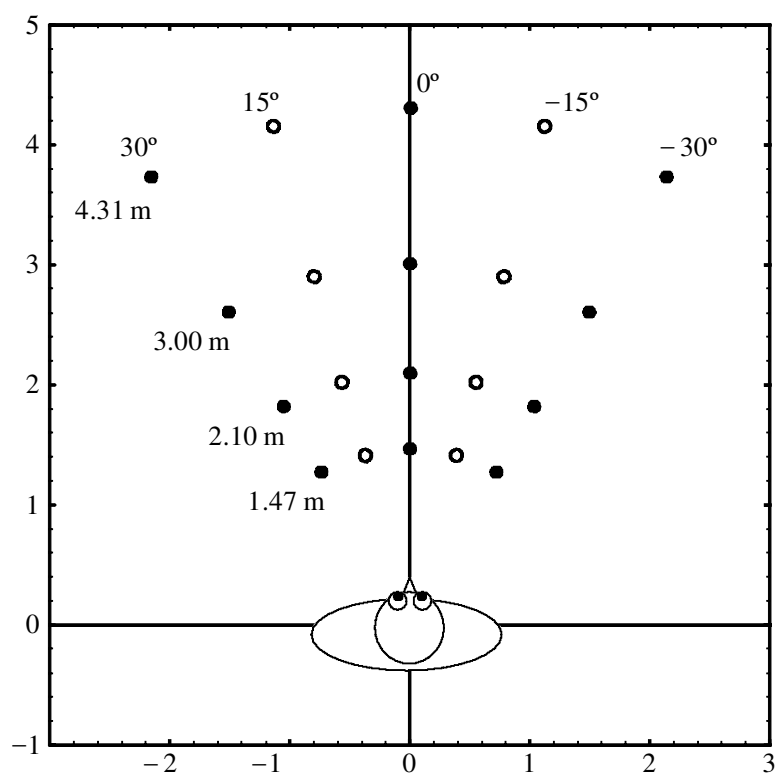

Figure 2. Schematic layout of the experimental room. The subject was located at the origin. The locations of the two bars were selected from either the positions indicated by the filled circles or the positions indicated by the open circles. As a result, the separation angle between the bars was either $30^{\circ}$ or $60^{\circ}$. 


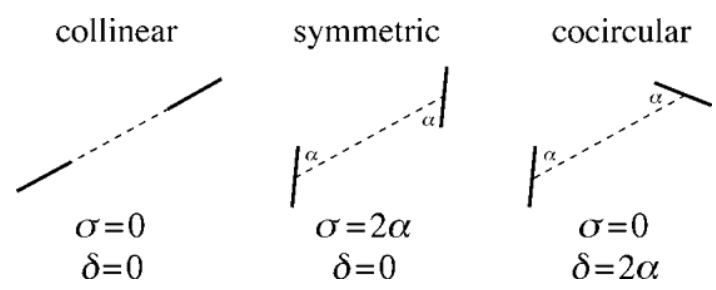

Figure 3. Values of the sum of the deviations $\sigma$ and the difference in deviations $\delta$ when the bars were physically collinear, symmetric, and cocircular.

Subject T.D. the average value is $18.1^{\circ} \pm 3.3^{\circ}$ (Figure 4a). An exception to this rule occurred for Subject R.F. when the bars were placed at equal distance from the subject (top left graph in Figure 4b). In this case, the difference between the orientations of the left and right bars is much less $\left(-11.6^{\circ} \pm 7.9^{\circ}\right)$ than for the other distance ratios $\left(9.0^{\circ} \pm\right.$ $\left.4.4^{\circ}\right)$, and it decreases with the radial distance. It turns out that this is the only exception and that the difference in orientation is approximately constant for all subjects and all combinations of angles at which the bars were placed (even for Subject R.F.).

As expected, the orientations of the left and right bars are not independent of the distance ratio. It is clear from Figure 4 that the orientations of both bars covaries with the veridical orientation indicated by the dashed line. More interestingly, the deviations from veridical, indicated in degrees below each bar, also depends systematically on the distance ratio: For both subjects the values increases from the second row down in the left column and decreases in the right column. In the frontoparallel condition (top row), the deviations are intermediate to those in the second row. By using the deviation sum $\sigma=\Delta \phi_{\text {left }}+\Delta \phi_{\text {right }}$ and the deviation difference $\delta=\Delta \phi_{\text {left }}-\Delta \phi_{\text {right }}$, the deviations were split into a symmetric part $\sigma$, which is the deviation from collinearity, and a mirror-symmetric part $\delta$, which corresponds to the deviation from parallelity. Since the orientations of both the left and right bars are very similar for a fixed distance ratio, this means that both the sum $\sigma$ and the difference $\delta$ are approximately constant for a fixed distance ratio. However, the sum $\sigma$ depends strongly on the distance ratio whereas the difference $\delta$ does not.

Figure 5 shows the results for Subjects T.D. and R.F. when the left and right bars were placed at $\psi_{\text {left }}=30^{\circ}$ and $\psi_{\text {right }}=0^{\circ}$, respectively. Otherwise, the layout is identical to Figure 4. Although the actual orientations of the bars differ from those shown in Figure 4 due to the different locations of the bars, the deviations are very similar for both subjects: Deviations of as much as $16^{\circ}$ occur, and the deviations of the left bar within each graph are very similar, as are those of the right bar. Moreover, the deviations of the left and right bars are also very similar to each other. In fact, the deviation difference $\delta$, corresponding to the deviation from parallel, is approximately zero and independent of the distances of the bars from the subject. For Subjects T.D. and R.F., the average values of $\delta$ are $-1.8^{\circ} \pm 4.2^{\circ}$ and $0.8^{\circ} \pm 3.1^{\circ}$, respectively. On the other hand, the sum of the deviations $\sigma$ depends strongly on the ratio between the distances of the bars from the subject: For each subject (panels a and b of Figure 5), the deviations of both bars increase from the second row down in the left column and decrease in the right column (similar to Figure 4). Again, the deviations in the top row (when the left and right bars were placed at equal distance from the subject) lie in between the deviations in the left and right graphs of the second row.

\section{Effect of the Distance}

In Figure 6 the difference in deviations is plotted as a function of the ratio between the distances of the left and right bars from the subject. The rows correspond to the subjects whose initials are denoted in the lower left corner of each graph, and the columns correspond to the different combinations of angles at which the left and right bars were placed. From left to right the combinations of angles are $\left(30^{\circ}, 0^{\circ}\right),\left(15^{\circ},-15^{\circ}\right),\left(0^{\circ},-30^{\circ}\right)$, and $\left(30^{\circ},-30^{\circ}\right)$, respectively. The different symbols correspond to the different radial distances of the left bar from the subject: The diamonds correspond to a distance of $1.47 \mathrm{~m}$, the stars to $2.1 \mathrm{~m}$, the squares to $3 \mathrm{~m}$, and the triangles to $4.31 \mathrm{~m}$. The error bars denote the standard deviations.

In each graph (Figure 6), the data points coincide quite well for the different distances of the left bar and lie approximately on a horizontal line. This means that, as noted previously, the deviation difference $\delta$ was mostly independent of the distance of the bars from the subject. Moreover, the data are in most cases close to zero, meaning that the bars are close to physically parallel. Two notable exceptions are visible for Subjects F.P. and T.D. in the fourth column: When the polar angles of the left and right bars are $30^{\circ}$ and $-30^{\circ}$, respectively, the average deviation difference $\delta$ is $17.4^{\circ}$ for Subject F.P. and $18.1^{\circ}$ for Subject T.D. The deviation difference $\delta$ is not always independent of the distance of the bars: In particular, for Subject R.F. in the fourth column, $\delta$ is much less at a distance ratio of 1 than for the other distance ratios and the data points do not coincide very well. In this case the bars were arranged in the frontoparallel plane and may be considered as tangent to a frontoparallel horopter. We will discuss this in more detail in the section on frontoparallel horopters.

Since the difference in deviations is approximately independent of the distances at which the bars were placed, it is useful to calculate the averages. In Table 2 the average values of the difference $\delta$ and their standard deviations are shown for each subject and each combination of angles. The average is taken over 48 trials consisting of three repetitions and four distances for each of the bars. The polar angles of the bars $\left(\psi_{\text {left }}, \psi_{\text {right }}\right)$ are shown in degrees above each column, and the rows correspond to the different subjects. The 95\% confidence intervals are obtained by multiplying the standard deviation by $t_{0.025} / \sqrt{n} \approx 0.53$ where $n=16$. It can be seen that in most cases the average difference is not significantly different from zero or is very close 
a)
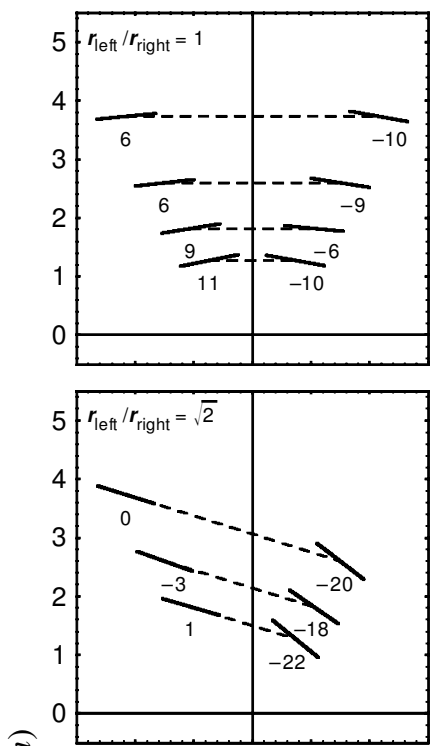

$\lesssim$
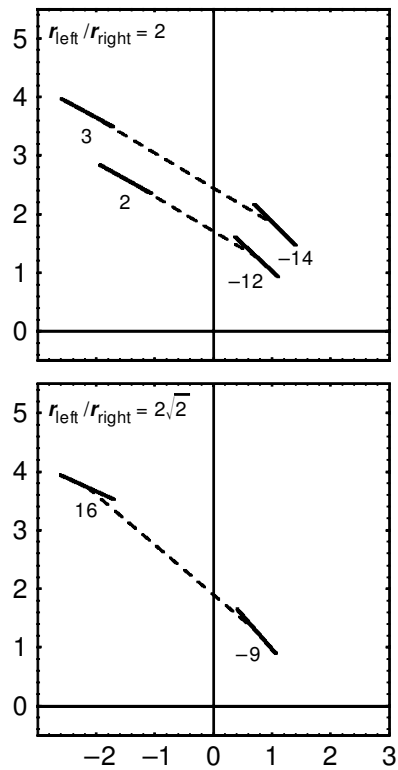
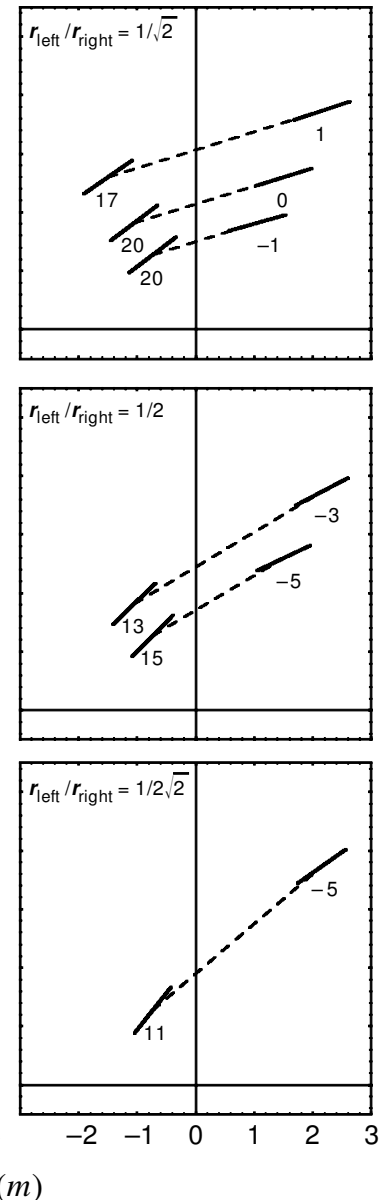

b)
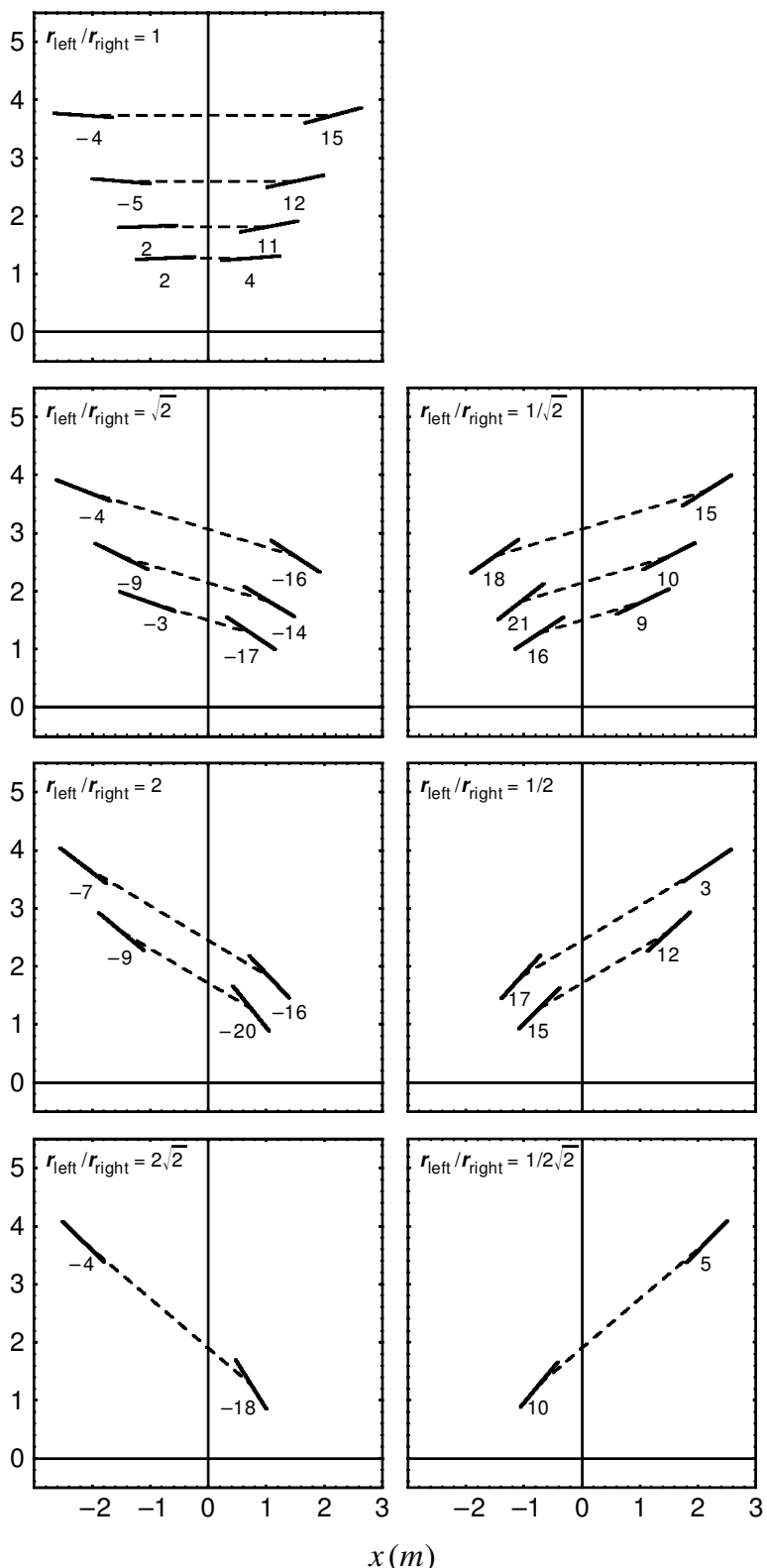

Figure 4. Graphical and numerical representation of the results for Subjects T.D. (panel a) and R.F. (panel b) when the left bar was located at $30^{\circ}$ and the right bar at $-30^{\circ}$. Each graph depicts the results for a fixed ratio between the distances of the left and right bars from the subject (indicated in the upper left corner). The location and orientation of each bar are indicated by a line and the veridical orientation is indicated by a dashed line. The deviation from veridical is shown numerically in degrees below each bar.

to zero. There are two exceptions for Subjects F.P. and T.D.- - namely when the left and right bars were positioned at angles of $30^{\circ}$ and $-30^{\circ}$, respectively.

The standard deviation is always about $4^{\circ}$. Thus, the standard deviation of half the difference, corresponding to the standard deviation for each bar individually, is $2^{\circ}$. This standard deviation can be compared directly to the standard deviation due to the three repetitions of each trial, which is about $2^{\circ}$ to $3^{\circ}$. Clearly, the order of magnitude is the same, which confirms the fact that the difference $\delta$ is in good approximation a constant. Apart from this global trend, an exception occurs for Subject R.F. when the left and right bar were positioned at angles of $30^{\circ}$ and $-30^{\circ}$, respectively (fourth column). From Figure $4 \mathrm{~b}$ it can be seen that the difference between the orientations of the left and right bars is much smaller when the radial distances from the observer were equal than when they were not. If we split the results into the frontoparallel cases and the non- 
a)
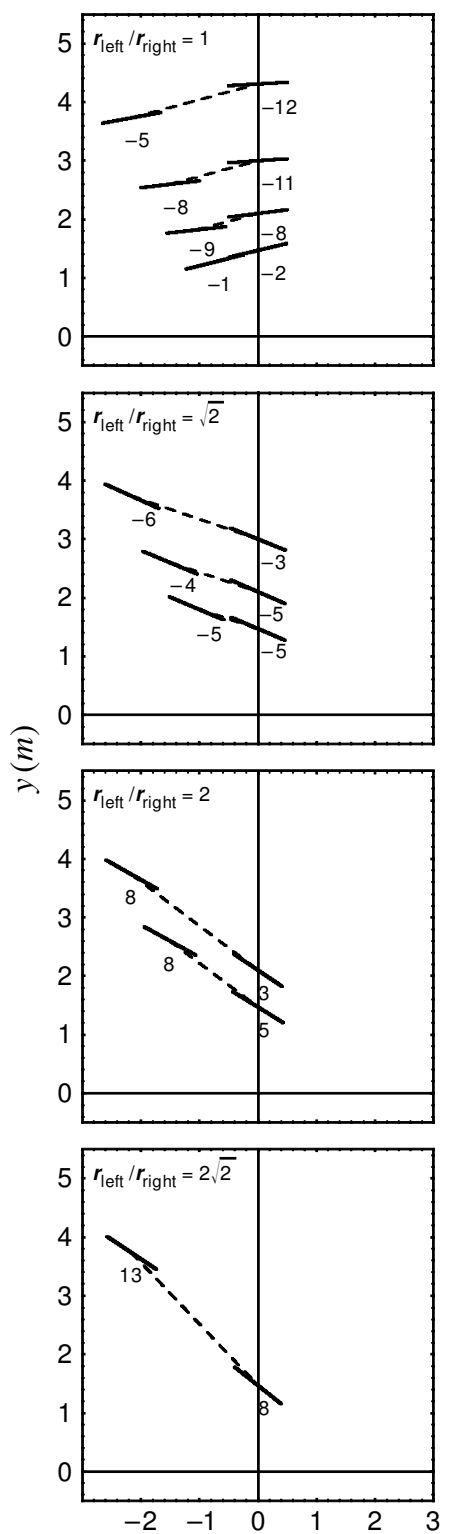

T.D. $\left(30^{\circ}, 0^{\circ}\right)$
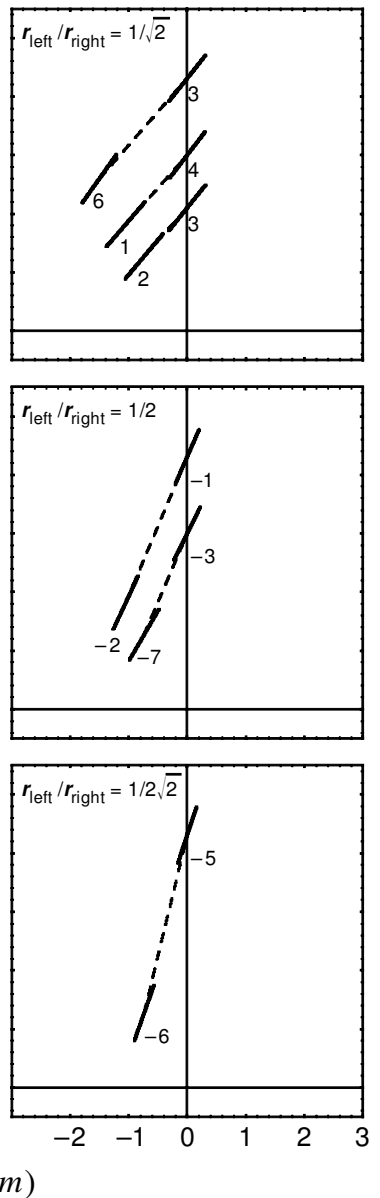

b)

R.F. $\left(30^{\circ}, 0^{\circ}\right)$
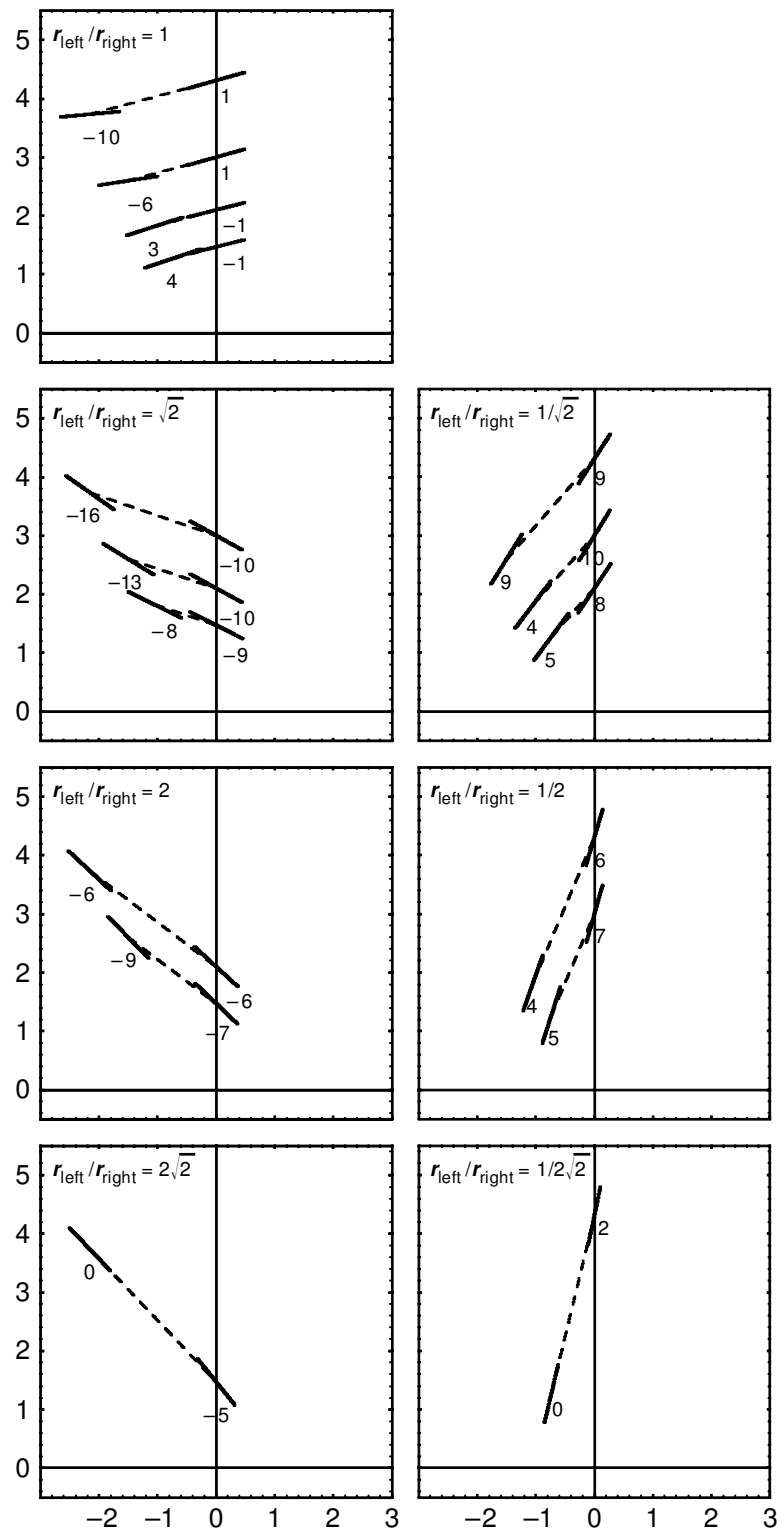

$x(m)$

Figure 5. Graphical and numerical representation of the results for Subjects T.D. (panel a) and R.F. (panel b) when the left bar was located at $30^{\circ}$ and the right bar at $0^{\circ}$. Each graph depicts the results for a fixed ratio between the distances of the left and right bars from the subject (indicated in the upper left corner). The location and orientation of each bar are indicated by a line and the veridical orientation is indicated by a dashed line. The deviation from veridical is shown numerically in degrees below each bar.

frontoparallel cases, we find that the average of the deviation difference was $-11.6^{\circ} \pm 7.9^{\circ}$ for the frontoparallel cases and $9.0^{\circ} \pm 4.4^{\circ}$ for the other cases. When this distinction is made, the standard deviations (divided by 2) are again comparable to the spread in the data due to the three repetitions.

The deviation difference $\delta$ reflects the mirror-symmetric part of the deviations of the left and right bars (Figure 3), where the mirror symmetry is with respect to the normal of the connecting line. In most cases the mirror-symmetric component is approximately zero; this means that the bars were physically parallel but not necessarily collinear.

The logical counterpart of the difference $\delta$ in the deviations of the left and right bars is the sum of the deviations $\sigma$. The deviation sum reflects the symmetric part of the deviations of the left and right bars (Figure 3). In Figure 7 the deviation sum $\sigma$ is plotted as a function of the ratio between the distances of the left and right bars 

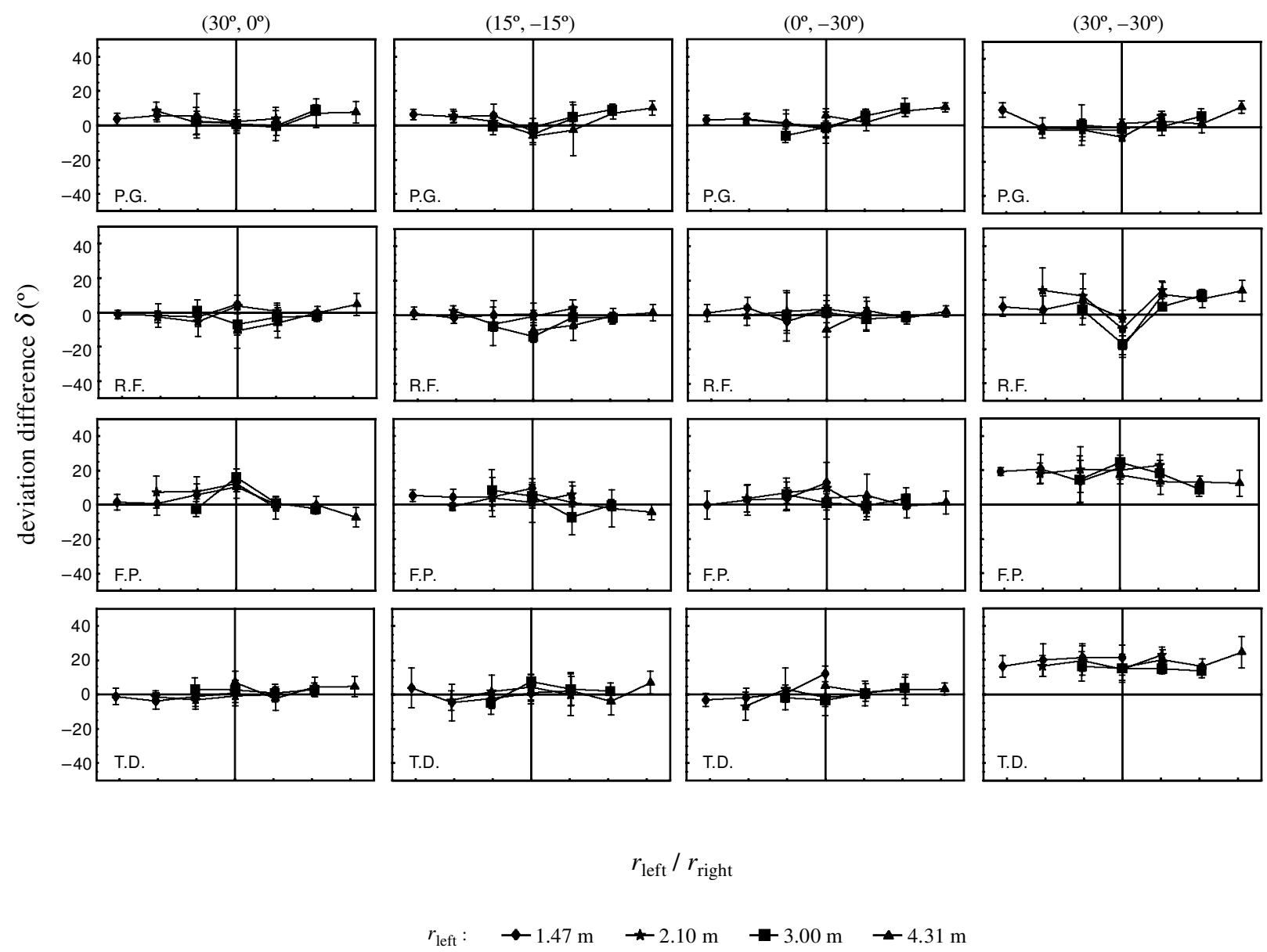

Figure 6. The difference $\delta$ between the deviations of the left and right bars as a function of the relative distance. The rows correspond to the different subjects, indicated in the lower left corner. The columns correspond to the different angles at which the left and right bars were placed: $\left(30^{\circ}, 0^{\circ}\right),\left(15^{\circ},-15^{\circ}\right),\left(0^{\circ},-30^{\circ}\right)$, and $\left(30^{\circ},-30^{\circ}\right)$, respectively. The diamond, square, triangle, and star symbols correspond to the distances of the left bar from the subject of $1.47,2.10,3.00$, and $4.31 \mathrm{~m}$, respectively.

from the subject. The layout and the meaning of the symbols are identical to those shown in Figure 6.

From Figure 7 it can be seen that, within each graph, there is a considerable overlap of the data. Since each line corresponds to a fixed distance of the left bar, this means that the influence of the absolute distances is negligible and that the measured deviations depend primarily on the relative distance $r_{\text {left }} / r_{\text {right }}$. Furthermore, the shape of the data as a function of the distance ratio $r_{\text {left }} / r_{\text {right }}$ is similar overall: The deviations first increase to a maximum (at a distance ratio of about $1 / \sqrt{2}$ ), after which they fall off steeply to a minimum (at a distance ratio of about $\sqrt{2}$ ) and then increase again. In most cases the deviation sum $\sigma$ is also approximately an antisymmetric function of the distance ratio. This means that the size of the deviation of each bar is interchanged and in the opposite direction when the distances at which the bars are placed are interchanged. In other words, the settings are symmetrical about the median plane. Typically, the deviations are positive for distance ratios smaller than 1 and negative for distance ratios larger than 1 . This means that the nearest bar tends to have an overshoot, whereas the furthest bar tends to have an undershoot (provided that deviation difference $\delta$ is sufficiently small). For very large and very small distance ratios, the bar locations were nearly aligned with the median line, in which case no significant deviations are expected. This may be the reason why the deviation sum $\sigma$ tends to zero for the largest and smallest distance ratios. The deviation sum $\sigma$ is not al-

Table 2

Mean and Standard Deviation of the Difference in Deviations $\left(\Delta \phi_{\text {left }}-\Delta \phi_{\text {right }}\right)$ for Each Subject

\begin{tabular}{|c|c|c|c|c|c|c|c|c|}
\hline \multirow[b]{2}{*}{ Subject } & \multicolumn{2}{|c|}{$\left(30^{\circ}, 0^{\circ}\right)$} & \multicolumn{2}{|c|}{$\left(15^{\circ},-15^{\circ}\right)$} & \multicolumn{2}{|c|}{$\left(0^{\circ},-30^{\circ}\right)$} & \multicolumn{2}{|c|}{$\left(30^{\circ},-30^{\circ}\right)$} \\
\hline & $M$ & $S D$ & $M$ & $S D$ & $M$ & $S D$ & $M$ & $S D$ \\
\hline P.G. & 3.6 & 3.2 & 2.5 & 5.1 & 3.1 & 4.6 & 2.0 & 4.7 \\
\hline R.F. & -1.8 & 4.2 & -2.3 & 4.4 & 0.2 & 3.3 & 3.8 & 10.6 \\
\hline F.P. & 3.7 & 6.5 & 2.6 & 4.7 & 3.6 & 4.1 & 17.4 & 4.3 \\
\hline T.D. & 0.8 & 3.1 & 0.9 & 3.9 & 0.9 & 4.3 & 18.1 & 3.3 \\
\hline
\end{tabular}

Note-Each pair of columns denotes the different angles in degrees at which the left and right bars are placed. 

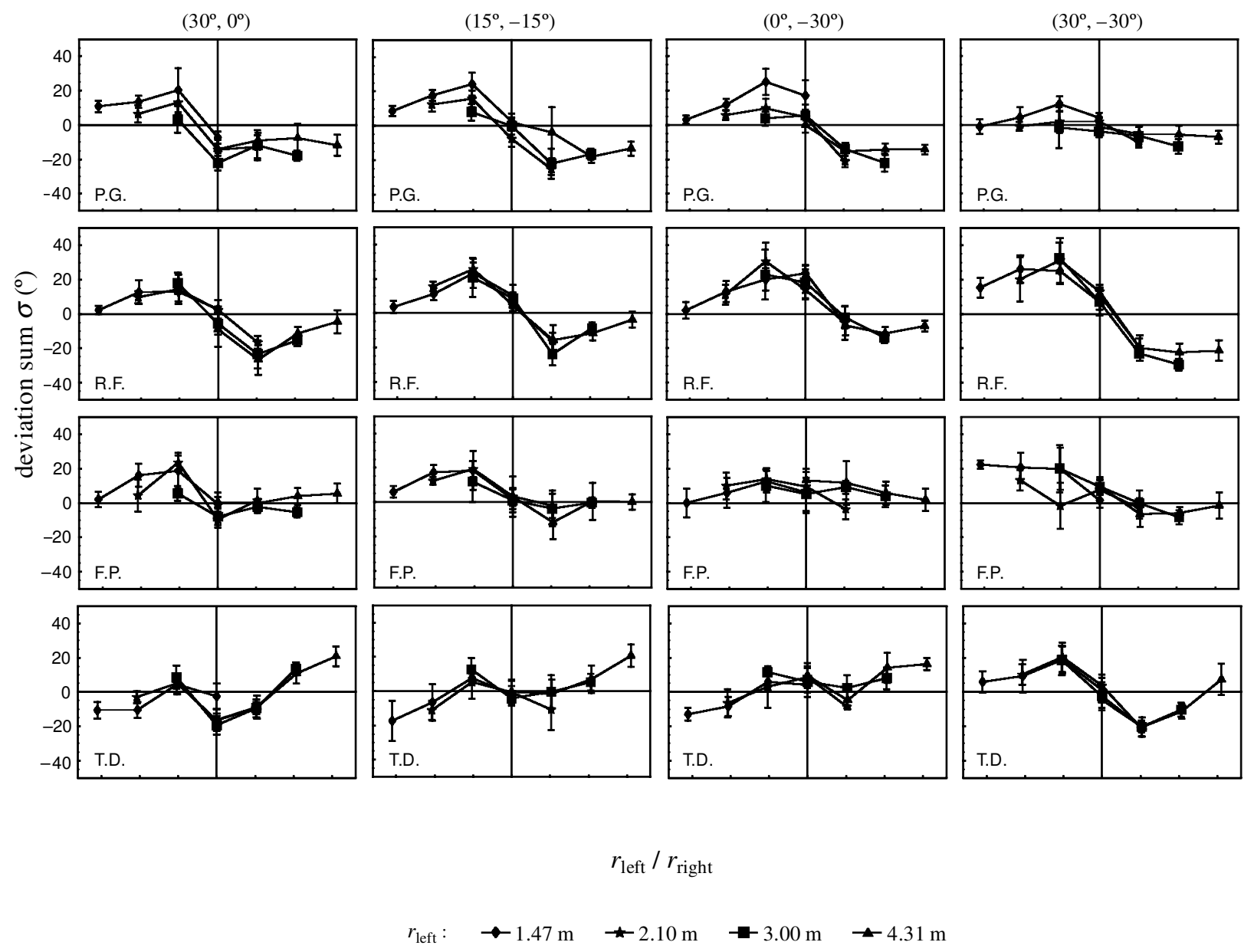

Figure 7. The sum $\sigma$ of the deviations of the left and right bars as a function of the relative distance. The rows correspond to the different subjects, indicated in the lower left corner. The columns correspond to the different angles at which the left and right bars were placed: $\left(30^{\circ}, 0^{\circ}\right),\left(15^{\circ},-15^{\circ}\right),\left(0^{\circ},-30^{\circ}\right)$, and $\left(30^{\circ},-30^{\circ}\right)$ respectively. The diamond, square, triangle, and star symbols correspond to the distances of the left bar from the subject of $1.47,2.10,3.00$, and $4.31 \mathrm{~m}$, respectively.

ways approximately antisymmetrical. This is most pronounced for Subject F.P.; see especially the third column of Figure 7. In that case $\sigma$ is approximately a symmetric function of the distance ratio.

\section{Effect of the Angular Displacement}

The similarities between the different columns in Figure 7 are striking, but some small differences can still be seen. In the first three columns, the separation angle has a constant value of $30^{\circ}$, but the positions of the bars are displaced $15^{\circ}, 0^{\circ}$, and $-15^{\circ}$ to the left, respectively. This angular displacement is equal to the bisecting angle, defined by $1 / 2\left(\psi_{\text {left }}+\psi_{\text {right }}\right)$. As can be seen from Figure 7 , there is hardly any systematic difference between the first three columns. Further analysis reveals that the only systematic effect of the angular displacement occurs when the left and right bars were placed at the same distance from the subject (distance ratio 1): For all subjects the deviations increase from the first column (angular displacement of $15^{\circ}$ ) in Figure 7 to the third column (angular displace- ment of $-15^{\circ}$ ). In the fourth column the angular displacement is $0^{\circ}$ and equal to the second column. For these two conditions $\left(\left[15^{\circ},-15^{\circ}\right]\right.$ and $\left.\left[30^{\circ},-30^{\circ}\right]\right)$, the values of $\sigma$ are very similar when the distance ratio is 1 . Thus, for a distance ratio of 1 , the deviation sum $\sigma$ increases as the angular displacement decreases, or, in other words, as the orientation of the configuration of the bars changes from left to right.

\section{Effect of the Separation Angle}

In the second and fourth column of Figure 7 the left and right bars were placed at symmetric angles with the median line, $\left(15^{\circ},-15^{\circ}\right)$ and $\left(30^{\circ},-30^{\circ}\right)$. In these cases the separation angles were $30^{\circ}$ and $60^{\circ}$, respectively, and the angular displacement was $0^{\circ}$. By correlating the values of the deviation sum $\sigma$ for a separation angle of $60^{\circ}$ with those for a separation of $30^{\circ}$, one can determine the first order effects: For Subject P.G. the size of the average deviations are scaled with a factor of about $0.3\left(R^{2}=\right.$ $.69)$, for Subject R.F. with a factor of $1.3\left(R^{2}=.84\right)$, and 
for Subject F.P. with a factor of $0.8\left(R^{2}=.42\right)$. The latter scaling factor does not significantly differ from 1 . For Subject T.D. the data does not correlate $\left(R^{2}=.06\right)$. This is due to the fact that the data for a separation angle of $30^{\circ}$ are sheared counterclock wise relative to the data for a separation angle of $60^{\circ}$ (Figure 7).

For Subjects F.P. and T.D., the separation angle has an effect on the average value of the deviation difference $\delta$ as well (Table 2): The mean value for a separation of $60^{\circ}$ (fourth column) is larger than that for a separation of $30^{\circ}$ (second column). However, no correlation is found between the values of the deviation difference $\delta$ for a separation angle of $60^{\circ}$ and those for a separation of $30^{\circ}$. Thus, the influence of the separation angle on $\delta$ is minor and very subject dependent.

\section{Frontoparallel Horopters}

For a small subset of the present results the bars were located in a (physically) frontoparallel plane. This is the case when the distance ratio equals 1 and when the bars are placed at symmetric angles with respect to the subject. Since many reports in the literature are concerned with apparent frontoparallel horopters, we will present our results in more detail for these conditions.

In Figure 8 the deviation sum $\sigma$ and the deviation difference $\delta$ are shown as a function of the distance for each subject when the bars were located in the frontoparallel plane. From top to bottom the results are shown for Subjects P.G., R.F., F.P., and T.D. From left to right the bars were located at the angles $\left(15^{\circ},-15^{\circ}\right)$ with a separation angle of $30^{\circ}$ and at $\left(30^{\circ},-30^{\circ}\right)$ with a separation angle of $60^{\circ}$. As can be seen, both $\sigma$ (stars) and $\delta$ (diamonds) are approximately independent of the distance. The only exception is Subject R.F., for whom $\delta$ decreases as a function of the distance for a separation of $60^{\circ}$ (right column) and only slightly for a separation of $30^{\circ}$ (left column). Because the bars were placed at mirror-symmetric positions about the median line, the deviation sum $\sigma$ should have been zero. This is true in most cases but not for Subject R.F., for whom the average deviation sum is $\sigma=10^{\circ} \pm 3^{\circ}$ for a separation of $60^{\circ}$ and $\sigma=7^{\circ} \pm 3^{\circ}$ for a separation of $30^{\circ}$. For Subject F.P. the same tendency is observed but only for a separation of $60^{\circ}\left(\sigma=7^{\circ} \pm 4^{\circ}\right)$. In summary, for Subjects P.G., T.D., and partly for Subject F.P., the settings are symmetrical about the median line, but not for Subject R.F.

In most cases the deviation difference is approximately zero, especially when the separation was $30^{\circ}$ (left column), but when the separation was $60^{\circ}$ (right column) there are several exceptions: For Subjects F.P. and T.D., the deviation difference is approximately $\delta=21^{\circ} \pm 3^{\circ}$ and $\delta=17^{\circ} \pm 3^{\circ}$, respectively, and for Subject R.F. the difference in deviations gradually decreases with the distance from $-2^{\circ}$ to $-19^{\circ}$.

When the deviation sum $\sigma$ is zero, the bars are tangent to a circular arc the curvature of which depends on the value of $\delta$. It is clear that since the difference $\delta$ is in most cases zero, the curvature of the corresponding horopter is also zero. However, for a separation angle of $60^{\circ}$ there are some exceptions: For Subject T.D. the deviation difference is on average $17^{\circ}$ when the separation was $60^{\circ}$. This corresponds to a curvature, concave toward the observer, of $\kappa=-0.3 / r$, where $r$ is the distance of the bars from the subject. For Subjects F.P. and R.F. the bar orientations were not mirror-symmetrical for a separation angle of $60^{\circ}$. If we ignore this and attend only to the mirror-symmetric part, we can find a measure for the curvature. For Subject F.P. this yields $\kappa=-0.4 / r$. For Subject R.F. the deviation difference $\delta$ is not approximately constant, so we will have to calculate the curvature for each distance separately: We find $0.02 \mathrm{~m}^{-1}, 0.07 \mathrm{~m}^{-1}, 0.10 \mathrm{~m}^{-1}$, and $0.08 \mathrm{~m}^{-1}$ for the distances of $1.47,2.10,3.00$, and $4.31 \mathrm{~m}$, respectively.

\section{DISCUSSION AND CONCLUSIONS}

We found that when subjects have to align two bars in the horizontal plane at eye height, the orientations of both bars depend systematically on the ratio between the distances of the bars from the subject. The deviations of the left and right bars can be split into two independent components. The first component is the sum $\sigma$ of the left and right deviations. We found that its value varies as a function of the ratio between the distances of the bars from the subject. Varying the distances while keeping the distance ratio constant has no significant effect. In first approximation the deviations are mirror-symmetric about the line bisecting the lines of gaze toward the left and right bars, which is to be expected from a geometrical point of view. This follows from the fact that when the left bar is placed at the distance of the right bar and vice versa, the deviation sum $\sigma$ changes sign. The effect of the separation angle on $\sigma$ is subject dependent but, except for Subject T.D., it did not influence the way the deviations depend on the distance ratio: The values of the deviation sum $\sigma$ for a separation of $60^{\circ}$ are scaled in size relative to those for a separation angle of $30^{\circ}$. We found scaling factors of 0.3 , 1.3, and 0.8 for Subjects P.G., R.F., and F.P., respectively. For Subject T.D., the values of $\sigma$ are sheared. No systematic effect of the angular displacement was observed except when the bars were placed at the same distance from the observer. In that case the deviation sum $\sigma$ increases as the orientation of the configuration of the bar positions changes from left to right.

Very similar results were found in a pointing task (Cuijpers, Kappers, \& Koenderink, 2000a) where subjects had to point to a target with a remotely controlled pointer in a very similar experimental setup: The pointing deviations showed the same pattern as the deviation sum $\sigma$; that is, they depended only on the distance ratio with an overshoot when the pointer was nearer than the target and vice versa. This indicates that the collinearity task is similar to pointing both bars at each other. Similar to the pointing task, the deviations can be related to a distorted distance perception. It can be shown that the distance function relating the perceived distance ratio to the physical distance ratio must have a expanding part for distance ratios smaller than 1 and 


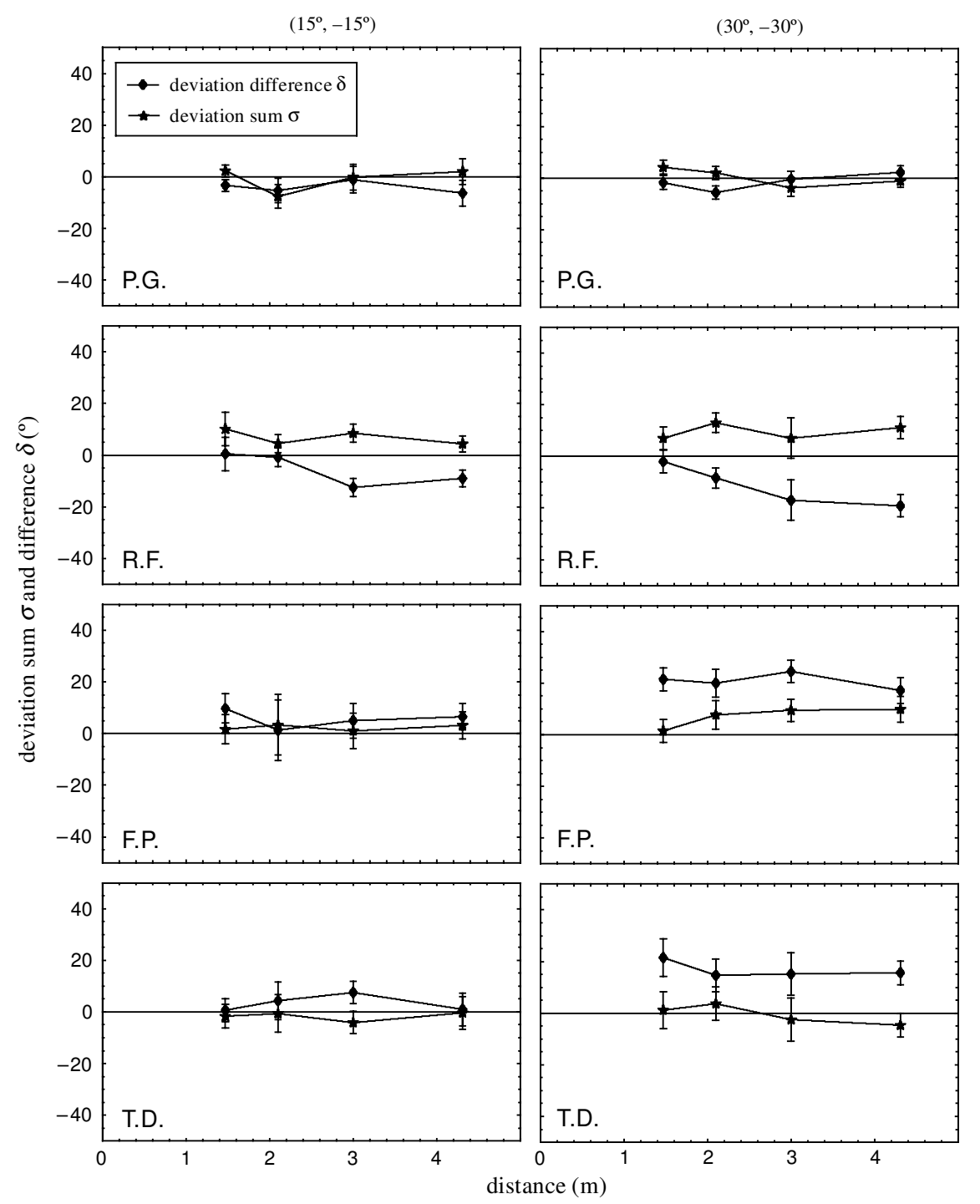

Figure 8. The deviation sum $\sigma$ and difference $\delta$ as a function of the distance when both bars were placed in the frontoparallel plane. The rows correspond to the different subjects, indicated in the lower left corner, and the columns correspond to the angles at which the bars were placed: $\left(15^{\circ},-15^{\circ}\right)$ and $\left(30^{\circ},-30^{\circ}\right)$.

a compressing part for distance ratios larger than 1 (see Cuijpers et al., 2000a, for details).

The second component is the difference $\delta$ between the deviations of the left and right bars. We found that the deviation difference $\delta$ is in good approximation a constant and independent of the positions of the left and right bars; the average difference is significantly different from zero only for Subjects F.P. and T.D. when the left and right bars were placed at $30^{\circ}$ and $-30^{\circ}$. However, in these cases the deviation difference $\delta$ is still independent of the distance of each bar from the subject. No effect of the angular displacement was observed.

If we assume that the bars are tangent to a visually straight line connecting the centers of the bars, our results can be compared to the (classical) frontoparallel horopter experiments (Battro et al., 1976; Helmholtz, 1962; Indow \& Watanabe, 1984a, 1988; Lukas, 1983; Shipley, 1959) when the bars were placed in the frontoparallel plane. It was found that for a separation angle of $30^{\circ}$ the deviation difference $\delta$ is approximately zero. As a consequence, the curvature of the corresponding circular arc is also zero. For a separation of $60^{\circ}, \delta$ is also zero for Subject P.G., but not for the other subjects. For Subjects T.D. and F.P. the values for $\delta$ are on average $17^{\circ}$ and $21^{\circ}$, respectively, which corresponds to a curvature of the frontoparallel horopters, concave toward the observer, of $\kappa=-0.3 / r$ and $\kappa=-0.4 / r$, respectively. For Subject R.F. the value of $\delta$ is not constant and is negative. The variability of the cor- 
responding curvatures is much smaller than for the other subjects; the average value is $\bar{\kappa}=0.06 \mathrm{~m}^{-1}$, which is convex toward the observer. These values are typically about an order of magnitude smaller than the values reported in the literature (see Shipley, 1959, for an overview). The settings of Subject R.F. were systematically different from symmetrical about the median line. Violations of this symmetry have been observed before (Battro et al., 1976; Foley, 1966; Ogle, 1964) and have been attributed to aniseikonia. It is not clear whether this is a valid explanation in the present case. In the literature several authors have reported an inversion from concave to convex at a distance of 1 or $2 \mathrm{~m}$. In our data the frontoparallel horopters were concave for Subjects T.D. and F.P. even at $4.31 \mathrm{~m}$ and convex for Subject R.F. No inversion point was observed, but this could be due to the limited distance range (1.47$4.31 \mathrm{~m})$. In general, the curvature of the horopters derived from our data was negligible or very small. Although our results disagree with some studies, the results in the literature are quite ambiguous: Sometimes an inversion point has been observed and sometimes not (Shipley 1959), and the reported curvatures vary considerably.

If visual space is Riemannian, collinear bars should also be parallel. As a consequence, the difference in orientation of the left and right bars in the collinearity task should be equal to the deviations found in the parallelity task reported earlier (Cuijpers et al., 2000b). In that study the same stimuli and stimulus positions were used, in the same environment. There, it was found that the deviations were independent of the distance and increased linearly with the separation angle. In particular, the mean deviation from parallelity was $7.0^{\circ}$ for a separation angle of $30^{\circ}$ and $18.7^{\circ}$ for a separation angle of $60^{\circ}$. In the present study the deviation difference $\delta$ (and thus the deviation from parallelity) was also independent of the distance. However, it was found that in most cases, the difference $\delta$ was approximately zero (Table 2 ). As a consequence, collinear bars do not, in general, appear to be parallel. This result is at odds with the assumption that visual space is isomorphic to some Riemannian space in which collinear vectors are always parallel. A possible explanation is that for the collinearity task the cues used differ from those used in the parallelity task. In the parallelity task the judgments could be based purely on the aspect ratio between the height and length of the bars. This is essentially a monocular cue, which would explain why the distance at which the bars are placed is irrelevant in the parallelity task. In the collinearity task, on the other hand, judgments of the aspect ratio of the bars are insufficient for performing the task correctly: Also the distances of the bars relative to the observer are needed. From the results it is clear that the subjects do indeed estimate the relative distance of the bars, albeit not veridically.

Although the different distance dependencies of the different tasks may be explained by the fact that the parallelity task can be performed monocularly, whereas the collinearity task cannot, it is hard to combine the results for both tasks in a single model. For example, if we as- sume that the locations of the bars are mapped to the same visual locations in the two tasks, then the intrinsic structure of visual space will differ depending on which task is used. Conversely, if it is assumed that the intrinsic structure of visual space is the same in the two tasks, then the positions of the bars must map to different visual positions depending on the task. However, normally the perceived object locations do not depend on the task in question. Therefore, we believe that the difference between the parallelity and collinearity tasks is indicative of a lack of consistency over different operationalizations of visual space. Such a lack of consistency was reported before for the perception of three-dimensional length (Norman, Lappin, \& Norman, 2000; Norman, Todd, Perotti, \& Tittle, 1996): It was found that the perceived distance could be both compressed and expanded, depending on the task. If it is the true that different tasks imply different operationalizations of visual space, then it will be necessary to reconsider the very concept of "visual space."

\section{REFERENCES}

Battro, A. M., Pierro Netto, S. Di, \& Rozestraten, J. A. (1976). Riemannian geometries of variable curvature in visual space: Visual alleys, horopters, and triangles in big open fields. Perception, 5, 9-23.

Battro, A. M., Reggini, H. C., \& Karts, C. (1978). Perspectives in open spaces: A geometrical application of the Thouless index. Perception, 7, 583-588.

BLANK, A. A. (1958). Axiomatics of binocular vision: The foundations of metric geometry in relation to space perception. Journal of the $\mathrm{Op}$ tical Society of America, 48, 328-334.

BLANK, A. A. (1961). Curvature of binocular visual space: An experiment. Journal of the Optical Society of America, 51, 335-339.

BLumenfeld, W. (1913). Untersuchungen über die scheinbare Grösse im Sehraume [Studies on apparent size in visual space). Zeitschrift für Psychologie, 65, 241-404.

Cuijpers, R. H., Kappers, A. M. L., \& Koenderink, J. J. (2000a). Investigation of visual space using an exocentric pointing task. Perception \& Psychophysics, 62, 1556-1571.

Cuijpers, R. H., Kappers, A. M. L., \& Koenderink, J. J. (2000b). Large systematic deviations in a visual parallelity task. Perception, 29, 1467-1482.

Cuijpers, R. H., Kappers, A. M. L., \& Koenderink, J. J. (2001). On the role of external reference frames on visual judgements of parallelity. Acta Psychologica, 108, 283-302.

FoLEY,J. M. (1963). Desarguesian property in visual space. Journal of the Optical Society of America, 54, 684-692.

Foley, J. M. (1966). Locus of perceived equidistance as a function of viewing distance. Journal of the Optical Society of America, 56, 822827.

Foley, J. M. (1972). The size-distance relation and intrinsic geometry of visual space: Implications for processing. Vision Research, 12, 323-332.

Foley, J. M. (1980). Binocular distance perception. Psychological Review, 87, 411-434.

GiBSON, J. J. (1966). The senses considered as perceptual systems. Boston: Houghton Mifflin.

GILINSKY, A. S. (1951). Perceived size and distance in visual space. Psychological Review, 58, 460-482.

HARDY, L. H., RAND, G., \& RITTLER, M. C. (1951). Investigation of visual space: The Blumenfeld alleys. Archives of Ophthalmology, 45, 53-63.

Helmholtz, H. von (1962). Helmholtz's treatise on physiological optics (J. P. C. Southall, Trans.). New York: Dover. (Original work published 1866)

Higashiy ama, A. (1984). Curvature of binocular visual space: A modified method of right triangle. Vision Research, 24, 1713-1718. 
Hillebrand, F. (1902). Theorie der scheinbaren Grösse bei binocularem Sehen [Theory of apparent size in binocular vision). Denkschriften der Wiener Akademie, Mathematisch-Naturwissenschaft Klasse, 72, 255-307.

InDOw, T. (1991). A critical review of Luneburg's model with regard to global structure of visual space. Psychological Review, 98, 430-453.

Indow, T., Inoue, E., \& Matsushima, K. (1962a). An experimental study of the Luneburg theory of binocular space perception (1): The 3- and 4-point experiments. Japanese Psychological Research, 4, 616.

Indow, T., Inoue, E., \& Matsushima, K. (1962b). An experimental study of the Luneburg theory of binocular space perception (2): The alley experiments. Japanese Psychological Research, 4, 17-24.

InDOW, T., \& Watanabe, T. (1984a). Parallel-alleys and distance-alleys on horopter plane in the dark. Perception, 13, 165-182.

Indow, T., \& Watanabe, T. (1984b). Parallel- and distance-alleys with moving points in the horizontal plane. Perception \& Psychophysics, 35, 144-154.

Indow, T., \& Watanabe, T. (1988). Alleys on an extensive apparent frontoparallel plane: A second experiment. Perception, 17, 647-666.

Koenderink, J. J., Van Doorn, A. J., \& Lappin, J. S. (2000). Direct measurement of curvature of visual space. Perception, 29, 69-79.

LUKAS, J. (1983). Visuelle Frontalparallelen: Ein Entscheidungsexperiment zu den Theorien von Blank, Foley und Luneburg [Visual frontoparallel horopters: A decision experiment between the theories of Blank, Foley and Luneburg). Zeitschrift für Experimentelle und Angewandte Psychologie, 30, 610-627.
Luneburg, R. K. (1947). Mathematical analysis of binocular vision. Princeton, NJ: Princeton University Press.

Norman, J. F., Lappin, J. S., \& Norman, H. F. (2000). The perception of length on curved and flat surfaces. Perception \& Psychophysics, 62, 1133-1145.

Norman, J. F., Todd, J. T., Perotti, V. J., \& Tittle, J. S. (1996). The visual perception of three-dimensional length. Journal of Experimental Psychology: Human Perception \& Performance, 22, 173-186.

OGLE, K. N. (1964). Researches in binocularvision. New York: Hafner. (Original work published 1950)

Schoumans, N., Koenderink, J. J., \& Kappers, A. M. L. (2000). Change in perceived spatial orientations due to context. Perception \& Psychophysics, 62, 532-539.

SHIPLEY, T. (1959). The frontal reference surfaces of visual space. Documenta Ophthalmalogica, 13, 487-516.

Stoker, J. J. (1969). Differential geometry. New York: WileyInterscience.

Wagner, M. (1985). The metric of visual space. Perception \& Psychophysics, 38, 483-495.

Walraven, J. (1975). Amblyopia screening with random-dot stereograms. Journal of Ophthalmology, 8, 178-182.

ZAJACZKOWSKA, A. (1956a). Experimental determination of Luneburg's constants $\sigma$ and K. Quarterly Journal of Experimental Psychology, 8 , 66-78.

ZaJAczKowsKa, A. (1956b). Experimental test of Luneburg's theory: Horopter and alley experiments. Journal of the Optical Society of America, 46, 514-527.

(Manuscript received July 24, 2000;

revision accepted for publication July 4, 2001.) 